PROCEEDINGS OF THE

AMERICAN MATHEMATICAL SOCIETY

Volume 133, Number 7 , Pages 2069-2076

S 0002-9939(05)07712-9

Article electronically published on January 31, 2005

\title{
AN EXTREMAL FUNCTION FOR THE CHANG-MARSHALL INEQUALITY OVER THE BEURLING FUNCTIONS
}

\author{
VALENTIN V. ANDREEV
}

(Communicated by Juha M. Heinonen)

\begin{abstract}
S.-Y. A. Chang and D. E. Marshall showed that the functional $\Lambda(f)=(1 / 2 \pi) \int_{0}^{2 \pi} \exp \left\{\left|f\left(e^{i \theta}\right)\right|^{2}\right\} d \theta$ is bounded on the unit ball $\mathcal{B}$ of the space $\mathcal{D}$ of analytic functions in the unit disk with $f(0)=0$ and Dirichlet integral not exceeding one. Andreev and Matheson conjectured that the identity function $f(z)=z$ is a global maximum on $\mathcal{B}$ for the functional $\Lambda$. We prove that $\Lambda$ attains its maximum at $f(z)=z$ over a subset of $\mathcal{B}$ determined by kernel functions, which provides a positive answer to a conjecture of Cima and Matheson.
\end{abstract}

\section{INTRODUCTION}

Let $\mathcal{D}$ be the Dirichlet space of functions $f$ analytic on the unit disk $\mathbb{D}$, with $f(0)=0$ and a finite Dirichlet integral

$$
\|f\|_{\mathcal{D}}^{2}=\frac{1}{\pi} \iint_{\mathbb{D}}\left|f^{\prime}(z)\right|^{2} d x d y .
$$

It is well known that $\mathcal{D}$ is a Hilbert space with inner product

$$
\langle f, g\rangle_{\mathcal{D}}=\frac{1}{\pi} \iint_{\mathbb{D}} f^{\prime}(z) \overline{g^{\prime}(z)} d x d y .
$$

Let $\mathcal{B}=\left\{f \in \mathcal{D}:\|f\|_{\mathcal{D}} \leq 1\right\}$ be its closed unit ball.

We shall be concerned with functionals $\Lambda_{\Phi}$ on $\mathcal{B}$ defined by

$$
\Lambda_{\Phi}(f)=\frac{1}{\pi} \int_{0}^{2 \pi} \Phi\left(\left|f\left(e^{i \theta}\right)\right|\right) d \theta
$$

for $f \in \mathcal{B}$ and $\Phi:(-\infty, \infty) \rightarrow \mathbb{R}$ being a continuous convex nondecreasing function. A function $f$ is a maximum for $\Lambda_{\Phi}$ if $f \in \mathcal{B}$ and $\Lambda_{\Phi}(f) \geq \Lambda_{\Phi}(g)$ for all $g \in \mathcal{B}$.

Chang and Marshall 3] proved that if $\Phi_{\alpha}(t)=e^{\alpha t^{2}}$ for $\alpha>0$, then $\Lambda_{\Phi_{\alpha}}$ is bounded on $\mathcal{B}$ if and only if $\alpha \leq 1$. In their proof they compared functions in $\mathcal{B}$ to the Beurling functions

$$
B_{a}(z)=\frac{\log \frac{1}{1-\bar{a} z}}{\sqrt{\log \frac{1}{1-|a|^{2}}}}
$$

Received by the editors August 1, 2003 and, in revised form, March 12, 2004.

2000 Mathematics Subject Classification. Primary 30H05; Secondary 30A10, 30D99, 49K99.

Key words and phrases. Dirichlet space, Chang-Marshall inequality, Baernstein star-function, extremal functions.

(C)2005 American Mathematical Society 2069

Reverts to public domain 28 years from publication 
for $a \in \mathbb{D} \backslash\{0\}$, where the branch of the logarithm is chosen so that $B_{a}(a)$ is real. The denominator assures that $\left\|B_{a}\right\|_{\mathcal{D}}=1$. Up to a normalizing factor, the $B_{a}$ are the kernel functions for $\mathcal{D}$. We shall denote by $\mathcal{B}_{0}$ the set of all Beurling functions and by $\widetilde{\mathcal{B}}_{0}$ its closed convex hull.

A shorter proof of this fact has since been found by Marshall [9]. A significantly more general and stronger inequality has been found by Essén [7. Andreev and Matheson [1] showed that the identity function $f(z)=z$ is a local maximum for $\Lambda_{\Phi_{1}}$ on $\mathcal{B}$ and conjectured that it is also a global maximum. Cima and Matheson 4] showed that the identity function is a local maximum on the set $\mathcal{B}_{0}$ and that the functional $\Lambda_{\Phi_{1}}$ attains its maximum on $\widetilde{\mathcal{B}}_{0}$. On the other hand, they showed that $\Lambda_{\Phi_{1}}$, when restricted to $\mathcal{B}$, is not weakly continuous at 0 , and thus it is an open question whether there exists a global maximum for $\Lambda_{\Phi_{1}}$ on $\mathcal{B}$. Matheson and Pruss [10] studied the regularity of the extremal functions. We refer the reader to their paper for an excellent discussion of this and other related problems and for a list of open problems.

Our principle result is:

Theorem 1.1. The inequality

$$
\Lambda_{\Phi_{1}}(f)<\Lambda_{\Phi_{1}}(z)
$$

holds true for all $f \in \widetilde{\mathcal{B}}_{0}$.

Our result proves Conjecture 1 of Cima and Matheson in [4].

\section{Proof of Theorem 1.1}

It is natural to set $B_{0}(z)=z$ (see 4]). A function $\Phi(x)$ continuous on $-\infty<$ $x<\infty$ is said to be convex if $\Phi((x+y) / 2) \leq[\Phi(x)+\Phi(y)] / 2$, and strictly convex if strict inequality holds whenever $x \neq y$. Theorem 1.1 is a consequence of the following result.

Theorem 2.1. Let $\Phi(x)$ be a convex nondecreasing function on $-\infty<x<\infty$. For all $a_{0}, a \in \mathbb{D} \backslash\{0\}$ such that $0 \leq\left|a_{0}\right|<|a|<1$, we have

$$
\int_{0}^{2 \pi} \Phi\left(\log \left|B_{a}\left(r e^{i \theta}\right)\right|\right) d \theta \leq \int_{0}^{2 \pi} \Phi\left(\log \left|B_{a_{0}}\left(r e^{i \theta}\right)\right|\right) d \theta,
$$

$0<r<1$. If $\Phi$ is strictly convex, then the inequality is strict for all $r$.

Proof. Our proof is based on the deep results of Albert Baernstein 2, Theorem 1] on integral means of univalent functions (see also Chapter 7 of Duren's book [5]). In particular, we need the following proposition [2, Proposition 3].

Proposition 2.2. For $g, h \in L^{1}(-\pi, \pi)$, the following statements are equivalent.

(a) For each function $\Phi(s)$ convex and nondecreasing on $-\infty<s<\infty$,

$$
\int_{-\pi}^{\pi} \Phi(g(x)) d x \leq \int_{-\pi}^{\pi} \Phi(h(x)) d x .
$$

(b) For each $t \in \mathbb{R}$,

$$
\int_{-\pi}^{\pi}[g(x)-t]^{+} d x \leq \int_{-\pi}^{\pi}[h(x)-t]^{+} d x .
$$

(c) $g^{*}(\theta) \leq h^{*}(\theta), 0 \leq \theta \leq \pi$. 
Here for each $r \in\left(r_{1}, r_{2}\right)$ and $u\left(r e^{i \theta}\right) \in L^{1}(0,2 \pi)$ the Baernstein star-function of $u$ is defined as

$$
u^{*}\left(r e^{i \theta}\right)=\sup _{|E|=2 \theta} \int_{E} u\left(r e^{i t}\right) d t
$$

$0 \leq \theta \leq \pi$, where $|E|$ denotes the Lebesgue measure of the set $E \subset[-\pi, \pi]$.

In view of Proposition 2.2, we want first to show that

$$
\int_{-\pi}^{\pi} \log ^{+}\left[\frac{\left|B_{a}\left(r e^{i \theta}\right)\right|}{\rho}\right] d \theta \leq \int_{-\pi}^{\pi} \log ^{+}\left[\frac{B_{a_{0}}\left(r e^{i \theta}\right)}{\rho}\right] d \theta,
$$

$0<r<1$, for each $\rho>0$ and all $a$ and $a_{0}$ such that $0 \leq\left|a_{0}\right|<|a|<1$. Notice that

$$
\int_{-\pi}^{\pi} \log ^{+}\left[\frac{\left|B_{a^{\prime}}\left(r e^{i \theta}\right)\right|}{\rho}\right] d \theta=\int_{-\pi}^{\pi} \log ^{+}\left[\frac{\left|B_{a^{\prime \prime}}\left(r e^{i \theta}\right)\right|}{\rho}\right] d \theta
$$

whenever $\left|a^{\prime}\right|=\left|a^{\prime \prime}\right|$. Hence we may assume from now on that $0 \leq a_{0}<a<0$.

We can apply Jensen's theorem to obtain

$$
\int_{-\pi}^{\pi} \log ^{+}\left[\frac{\left|B_{a}\left(r e^{i \theta}\right)\right|}{\rho}\right] d \theta=\int_{-\pi}^{\pi} N\left(r, \rho e^{i \phi}\right) d \phi,
$$

since $B_{a}(0)=0$. It is easy to see that $B_{a}$ is a univalent function in the unit disk $\mathbb{D}$, $B_{a}(0)=0$ and $B_{a}^{\prime}(0)=a / A$, where $A=\left\{\log \left[1 /\left(1-|a|^{2}\right)\right]\right\}^{1 / 2}$, for each $0<a<1$, with a continuous extension to the closed unit disk $\overline{\mathbb{D}}$, and if $\alpha=\rho e^{i \phi} \neq 0$ is in the range $D_{a}$ of $B_{a}$, then

$$
N(r, \alpha)=\int_{0}^{r} \frac{n(t, \alpha)}{t} d t=\log ^{+}\left[\frac{r}{|\alpha|}\right]=\log ^{+}\left[\frac{r}{\left|B_{a}^{-1}(\alpha)\right|}\right],
$$

$0<r<1$. Let $u_{a}(\zeta)=-\log \left|B_{a}^{-1}(\zeta)\right|$ be the Green's function of $D_{a}$ with pole at 0 . Extend it to a continuous function in the punctured plane by setting $u_{a}(\zeta)=0$, $\zeta \notin D_{a}$. The formula (2.5) takes the form

$$
N(r, \zeta)=\left[u_{a}(\zeta)+\log r\right]^{+},
$$

$0<r<1$, for arbitrary $\zeta$, and equation (2.4) becomes

$$
\int_{-\pi}^{\pi} \log ^{+}\left[\frac{\left|B_{a}\left(r e^{i \theta}\right)\right|}{\rho}\right] d \theta=\int_{-\pi}^{\pi}\left[u_{a}\left(\rho e^{i \phi}\right)+\log r\right]^{+} d \phi .
$$

Let $u_{a_{0}}(\zeta)=-\log \left|B_{a_{0}}^{-1}(\zeta)\right|$ for $\zeta \in D_{a_{0}}$, and let $u_{a_{0}}(\zeta)=0$ elsewhere. In view of (2.6) the inequality (2.3) can be recast in the form

$$
\int_{-\pi}^{\pi}\left[u_{a}\left(\rho e^{i \phi}\right)+\log r\right]^{+} d \phi \leq \int_{-\pi}^{\pi}\left[u_{a_{0}}\left(\rho e^{i \phi}\right)+\log r\right]^{+} d \phi,
$$

$0<r<1,0<\rho<\infty$. By Proposition 2.2, this is implied by the inequality

$$
u_{a}^{*}\left(\rho e^{i \phi}\right) \leq u_{a_{0}}^{*}\left(\rho e^{i \phi}\right)
$$

$0<\rho<\infty, 0 \leq \phi \leq \pi$.

The function $u(\zeta)$ is continuous in $0<|\zeta|<\infty$, it is positive and harmonic in $D_{a}$, and identically zero outside $D_{a}$. Thus it is subharmonic in $0<|\zeta|<\infty$. Hence by [2, Theorem A] and the definition (2.2) of the star-function, $u_{a}^{*}$ is subharmonic in the open upper half-plane and continuous in the closed upper half-plane, except at the origin. 
Since $B_{a}^{-1}(\zeta)=\left(1-e^{-A \zeta}\right) / a$, then, near the origin, $u_{a}$ has the form

$$
u_{a}(\zeta)=-\log |\zeta|-\log \frac{A}{a}+u_{1 a}(\zeta),
$$

where $u_{1 a}$ is harmonic and $u_{1 a}(0)=0$. Thus

$$
u_{a}^{*}\left(\rho e^{i \phi}\right)+2 \phi \log \rho \rightarrow-2 \phi \log \frac{A}{a}
$$

as $\rho \rightarrow 0$ for $0 \leq \phi \leq \pi$. Similarly, near the origin, $u_{a_{0}}$ has the form

$$
u_{a_{0}}(\zeta)=-\log |\zeta|-\log \frac{A_{0}}{a_{0}}+u_{1 a_{0}}(\zeta),
$$

where $u_{1 a_{0}}$ is harmonic and $u_{1 a_{0}}(0)=0$. Thus

$$
u_{a_{0}}^{*}\left(\rho e^{i \phi}\right)+2 \phi \log \rho \rightarrow-2 \phi \log \frac{A_{0}}{a_{0}}
$$

as $\rho \rightarrow 0$ for $0 \leq \phi \leq \pi$. It follows that

$$
\left[u_{a}^{*}\left(\rho e^{i \phi}\right)-u_{a_{0}}^{*}\left(\rho e^{i \phi}\right)\right] \rightarrow-2 \phi \log \frac{a_{0} A}{a A_{0}}
$$

as $\rho \rightarrow 0$ for $0 \leq \phi \leq \pi$. It is easy to see that $a_{0} A /\left(a A_{0}\right)>1$ for $a_{0}<a$ and hence that $-2 \pi \log \frac{a_{0} A}{a A_{0}} \leq-2 \phi \log \frac{a_{0} A}{a A_{0}} \leq 0$ for $a_{0}<a$.

Hence $\left(u_{a}^{*}-u_{a_{0}}^{*}\right)$ is subharmonic in the upper half-plane and continuous in its closure except at the origin, where it has a bounded discontinuity: for $\phi=0$,

$$
\lim _{\rho \rightarrow 0}\left(u_{a}^{*}(\rho)-u_{a_{0}}^{*}(\rho)\right)=0,
$$

and for $\phi=\pi$,

$$
\lim _{\rho \rightarrow 0}\left(u_{a}^{*}(-\rho)-u_{a_{0}}^{*}(-\rho)\right)=-2 \pi \log \frac{a_{0} A}{a A_{0}} .
$$

We want to show that $\left(u_{a}^{*}-u_{a_{0}}^{*}\right)<0$ in the open upper half-plane. Since $u_{a}^{*}-u_{a_{0}}^{*}$ is discontinuous at the origin, we cannot apply the maximum principle for subharmonic functions to $u_{a}^{*}-u_{a_{0}}^{*}$ at this point. The proof of the inequality $\left(u_{a}^{*}-u_{a_{0}}^{*}\right)<0$ for $\Im \zeta>0$ will be based on the following four steps (a)-(d).

(a) On the positive real axis, by definition, $u_{a}^{*}(\zeta)=v^{*}(\zeta)=0$ for $\zeta>0$.

(b) Next let $d_{a}$ be the distance from 0 to the complement of $D_{a}$. It is obvious that $\Re\left(1-a e^{i \theta}\right)^{-1}>0$. Since the branch of the logarithm was chosen so that $B_{a}(a)$ is real, then

$$
\left|B_{a}\left(e^{i \theta}\right)\right|=\frac{1}{A}\left\{\left[\log \frac{1}{\left|1-a e^{i \theta}\right|}\right]^{2}+\left[\arg \frac{1}{1-a e^{i \theta}}\right]^{2}\right\}^{1 / 2} .
$$

Since $\max \left|1-a e^{i \theta}\right|=\left|1-a e^{i \pi}\right|=1+a$ and $\left[\arg \frac{1}{1-a e^{i \pi}}\right]^{2}=0$, it is easy to see that

$$
-\frac{1}{A} \log \frac{1}{1+a} \leq\left|B_{a}\left(e^{i \theta}\right)\right| \leq \frac{1}{A} \log \frac{1}{1-a}
$$

for $0<a<1$. Thus $d_{a}=-\frac{1}{A} \log \frac{1}{1+a}$. We want to show that $d_{a}$ is a decreasing function of $a$ for $0<a<1$. It is clear that $d_{a} \rightarrow 1$ as $a \rightarrow 0$. Let

$$
f(a)=\frac{\log (1+a)}{A} \text {. }
$$

Then

$$
f^{\prime}(a)=-\frac{[(1-a) \log (1-a)+\log (1+a)]}{\left(1-a^{2}\right) A^{3}} .
$$


Let

$$
f_{1}(a)=(1-a) \log (1-a)+\log (1+a) .
$$

An easy computation shows that $f_{1}^{\prime \prime}(a)>0$ for $0<a<1$. Thus $f_{1}^{\prime}$ is an increasing function of $a$, and it follows that $f_{1}^{\prime}(a)>0$ for $0<a<1$ since $f_{1}^{\prime}(0)=0$. Therefore $f_{1}$ is an increasing function of $a$ for $0<a<1$ and $f_{1}(a)>0$ since $f_{1}(0)=0$. Finally, this implies that $f^{\prime}(a)<0$ for $0<a<1$, and thus $f$ is a decreasing function of $a$. Therefore $d_{a_{0}}>d_{a}$ for all $a, a_{0}<a<1$.

In the disk $|\zeta|<d_{a}, u_{a}(\zeta)$ has the form (2.8), where $u_{1 a}$ is harmonic in $|\zeta|<d_{a}$ and $u_{1 a}(0)=0$. Thus

$$
u_{a}^{*}\left(\rho e^{i \pi}\right)=-2 \pi \log \frac{1}{\rho}-2 \pi \log \frac{A}{a}
$$

and, similarly,

$$
u_{a_{0}}^{*}\left(\rho e^{i \pi}\right)=-2 \pi \log \frac{1}{\rho}-2 \pi \log \frac{A_{0}}{a_{0}}
$$

for $0<\rho<d_{a}$. Hence $u_{a}^{*}(\zeta)<u_{a_{0}}^{*}(\zeta)$ for $-d_{a} \leq \zeta<0$.

(c) Since $u_{1 a}(\zeta)$ and $u_{1 a_{0}}(\zeta)$ are harmonic in $|\zeta|<d_{a}$ and $u_{1 a}(0)=u_{1 a_{0}}(0)=0$, then for every $\epsilon>0$ there is a $\rho_{0}, \rho_{0}=\left|\zeta_{0}\right|<d_{a}$, such that $\left|u_{1 a}(\zeta)\right|<\epsilon / 2$ and $\left|u_{1 a_{0}}(\zeta)\right|<\epsilon / 2$ for all $\zeta,|\zeta| \leq \rho_{0}$. Thus

$$
\begin{aligned}
u_{a}^{*}\left(\rho e^{i \phi}\right) & =\sup _{|E|=2 \phi} \int_{E} u_{a}\left(\rho e^{i t}\right) d t \\
& =-2 \phi \log \rho-2 \phi \log \frac{A}{a}+\sup _{|E|=2 \phi} \int_{E} u_{1 a}\left(\rho e^{i t}\right) d t \\
& \leq-2 \phi \log \rho-2 \phi \log \frac{A}{a}+\phi \epsilon
\end{aligned}
$$

and

$$
\begin{aligned}
u_{a_{0}}^{*}\left(\rho e^{i \phi}\right) & =\sup _{|E|=2 \phi} \int_{E} u_{a_{0}}\left(\rho e^{i t}\right) d t \\
& =-2 \phi \log \rho-2 \phi \log \frac{A_{0}}{a_{0}}+\sup _{|E|=2 \phi} \int_{E} u_{1 a_{0}}\left(\rho e^{i t}\right) d t \\
& \geq-2 \phi \log \rho-2 \phi \log \frac{A_{0}}{a_{0}}-\phi \epsilon
\end{aligned}
$$

for $0<\rho \leq \rho_{0}$ and $0<\phi<\pi$. Now choose $\epsilon$ such that $\epsilon<\log \left(A a_{0} / a A_{0}\right)$. Then

$$
u_{a}^{*}\left(\rho e^{i \phi}\right)-u_{a_{0}}^{*}\left(\rho e^{i \phi}\right) \leq-2 \phi \log \frac{A a_{0}}{a A_{0}}+2 \phi \epsilon<0
$$

for all $0<\rho \leq \rho_{0}$ and $0<\phi<\pi$. Hence $u_{a}^{*}(\zeta)<u_{a_{0}}^{*}(\zeta)$ for $|\zeta| \leq \rho_{0}<d_{a}$ and $0<\phi<\pi$.

(d) To establish the inequality on $-\infty<\zeta<-d_{a}$, we fix $\epsilon>0$ and consider the function

$$
Q(\zeta)=u_{a}^{*}(\zeta)-u_{a_{0}}^{*}(\zeta)-\epsilon \phi
$$

$\zeta=\rho e^{i \phi}$, which is subharmonic in $\mathcal{A}=\left\{\zeta: \rho_{0}<|\zeta|, 0 \leq \Im \zeta\right\}$ and continuous in the closure of $\mathcal{A}$. Let $M$ be the maximum of $Q(\zeta)$ in $\overline{\mathcal{A}}$. Then $M \geq 0$ and, according to the maximum principle for subharmonic functions, the maximum is attained somewhere on the boundary of $\mathcal{A}$. Suppose $M>0$. Since $u_{a}^{*}(\zeta) \leq u_{a_{0}}^{*}(\zeta)$ on the set $\left\{\zeta:-d_{a} \leq \zeta \leq \rho_{0}\right\} \cup\left\{\zeta:|\zeta|=\rho_{0}, \Im \zeta>0\right\} \cup\left\{\zeta: \rho_{0} \leq \zeta<\infty\right\}$, there 
is some point $-\zeta_{1}=-\rho_{1}$ for which $-\infty<\zeta_{1}<-d_{a}$ and $Q\left(\zeta_{1}\right)=M$. Let $G_{a}(\phi)$ denote the symmetric decreasing rearrangement of $u_{a}\left(\rho_{1} e^{i \phi}\right)$. Then

$$
\frac{\partial u_{a}^{*}}{\partial \phi}\left(\rho_{1} e^{i \phi}\right)=2 G_{a}(\phi)
$$

for $0 \leq \phi \leq \pi$ by [2, Proposition 2]. But because $\rho_{1}>d_{a}$, there is some point on the circle $|\zeta|=\rho_{1}$ that lies outside $D_{a}$, so

$$
G_{a}(\pi)=\inf _{0 \leq \phi \leq \pi} u_{a}\left(\rho_{1} e^{i \phi}\right)=0 .
$$

Applying the same argument to $u_{a_{0}}^{*}$ we obtain

$$
\frac{\partial u_{a_{0}}^{*}}{\partial \phi}\left(\rho_{1} e^{i \phi}\right)=2 G_{a_{0}}(\phi)
$$

for $0 \leq \phi \leq \pi$. If $d_{a}<\rho_{1} \leq d_{a_{0}}$, then

$$
G_{a_{0}}(\phi)=\inf _{0 \leq \phi<\pi}\{t: \lambda(t) \leq 2 \phi\},
$$

where $\lambda$ is the distribution function of $u_{a_{0}}, \lambda(t)=\left|\left\{\phi: u_{a_{0}}\left(\rho_{0} e^{i \phi}\right)>t\right\}\right|$, and

$$
G_{a_{0}}(\pi)=\lim _{\phi \rightarrow \pi^{-}} G_{a_{0}}(\phi) .
$$

Hence $G_{a_{0}}(\pi) \geq 0$ if $d_{a}<\rho_{1} \leq d_{a_{0}}$. If $d_{a_{0}}<\rho_{1}$, there is some point on the circle $|\zeta|=\rho_{1}$ that lies outside $D_{a_{0}}$, so

$$
G_{a_{0}}(\pi)=\inf _{0 \leq \phi \leq \pi} u_{a_{0}}\left(\rho_{1} e^{i \phi}\right)=0 .
$$

Therefore

$$
\frac{\partial Q}{\partial \phi}\left(\zeta_{1}\right) \leq-\epsilon<0,
$$

which contradicts the assumption that $Q(\zeta)$ has a relative maximum at $\zeta_{1}$. Hence $M=0$ and

$$
u_{a}^{*}(\zeta) \leq u_{a_{0}}^{*}(\zeta)+\epsilon \phi \leq u_{a_{0}}^{*}(\zeta)+\epsilon \pi
$$

for $\zeta \in \overline{\mathcal{A}}$. Letting $\epsilon \rightarrow 0$ we obtain that

$$
u_{a}^{*}\left(\rho e^{i \phi}\right) \leq u_{a_{0}}^{*}\left(\rho e^{i \phi}\right)
$$

for $\zeta \in \overline{\mathcal{A}}$.

We are in a position now to prove that $u_{a}^{*}(\zeta)<u_{a_{0}}^{*}(\zeta)$ in the open upper halfplane. Combining (a) $-(\mathrm{d})$ we obtain (2.7). Furthermore, $u_{a}^{*}(\zeta)<u_{a_{0}}^{*}(\zeta)$ on the set $\left\{\zeta:-d_{a} \leq \zeta \leq \rho_{0}\right\} \cup\left\{\zeta:|\zeta|=\rho_{0}, \Im \zeta>0\right\}$ by (b) and (c). Hence $u_{a}^{*}-u_{a 0}^{*}$ is a subharmonic function on $\mathcal{A}$ that is not identically equal to zero there and, by the maximum principle, this implies that $u_{a}^{*}(\zeta)<u_{a_{0}}^{*}(\zeta)$ everywhere in $\mathcal{A}$. Also, $u_{a}^{*}(\zeta)<u_{a_{0}}^{*}(\zeta)$ for $\left\{\zeta: 0<|\zeta| \leq \rho_{0}<d_{a}, 0<\Im \zeta\right\}$ by (c). Therefore,

$$
u_{a}^{*}(\zeta)<u_{a_{0}}^{*}(\zeta)
$$

in the open upper half-plane.

It follows from Proposition 2.2 that

$$
\int_{0}^{2 \pi} \Phi\left(\log \left|B_{a}\left(r e^{i \theta}\right)\right|\right) d \theta \leq \int_{0}^{2 \pi} \Phi\left(\log \mid B_{a_{0}}\left(r e^{i \theta} \mid\right) d \theta\right.
$$

for all $0 \leq a_{0}<a<0$ and $0<r<1$. The proof of strict inequality in (2.9) is identical to the proof of strict inequality in Theorem 1 in [2, pp. 157-158] and will be omitted. This completes the proof of Theorem 2.1. 
Proof of Theorem 1.1. The choice $\Phi(x)=e^{e^{2 x}}$ in (2.1) allows us to conclude that

$$
\Lambda_{\Phi_{1}}\left(B_{a}\left(r e^{i \theta}\right)\right)<\Lambda_{\Phi_{1}}\left(B_{a_{0}}\left(r e^{i \theta}\right)\right)
$$

for all $0 \leq a_{0}<a<0$ and $0<r<1$. Let

$$
\left\|B_{a}\left(r e^{i \theta}\right)\right\|_{p}^{p}=\frac{1}{2 \pi} \int_{0}^{2 \pi}\left|B_{a}\left(r e^{i \theta}\right)\right|^{p} d \theta .
$$

Since

$$
\Lambda_{\Phi_{1}}\left(B_{a}\left(r e^{i \theta}\right)\right)=1+\sum_{n=1}^{\infty} \frac{\left\|B_{a}\left(r e^{i \theta}\right)\right\|_{2 n}^{2 n}}{n !}
$$

and, by Lemma 1 of [1], $B_{a} \in H^{p}$ for $0<p<\infty$, we can choose a sequence $r_{n} \rightarrow 1$ as $n \rightarrow \infty$ for which the inequalities $\Lambda_{\Phi_{1}}\left(B_{a}\left(r_{n} e^{i \theta}\right)\right)<\Lambda_{\Phi_{1}}\left(B_{a_{0}}\left(r_{n} e^{i \theta}\right)\right)$ hold. Hence

$$
\Lambda_{\Phi_{1}}\left(B_{a}\left(r e^{i \theta}\right)\right) \leq \Lambda_{\Phi_{1}}\left(B_{a_{0}}\left(r e^{i \theta}\right)\right)
$$

for all $0<r \leq 1$ by Hardy's convexity theorem for integral means (see, e.g., [6] Theorem 1.5]).

It now remains to demonstrate that strict inequality holds true in Theorem 1.1. According to Theorem 2 of [4, $B_{0}$ is a local maximum on the set of Beurling functions. Thus there is an $a_{0}, 0<a_{0}$, such that

$$
\Lambda_{\Phi_{1}}\left(B_{a}\left(e^{i \theta}\right)\right)<\Lambda_{\Phi_{1}}\left(B_{0}\left(e^{i \theta}\right)\right)
$$

for $0<a \leq a_{0}$. (James and Matheson [8] have informed the author that, using a numerical method, they have proved the last inequality for $0<a<1 / 2$.)

Finally, combine the last inequality with the fact that $\Lambda_{\Phi_{1}}$ is log-convex 4 , p. 387 ] to complete the proof of Theorem 1.1.

It was pointed out in [1] that $B_{0}$ does not maximize the integral means over $\mathcal{B}$. If we choose $\Phi(x)=e^{p x}, 0<p<\infty$, in Theorem 2.1, we obtain that $B_{0}$ maximizes the integral means over $\mathcal{B}_{0}$.

Corollary 2.3. The inequality

$$
\frac{1}{2 \pi} \int_{0}^{2 \pi}\left|B_{a}\left(r e^{i \theta}\right)\right|^{p} d \theta \leq \frac{1}{2 \pi} \int_{0}^{2 \pi}\left|B_{a_{0}}\left(r e^{i \theta}\right)\right|^{p} d \theta
$$

holds true for all $0 \leq\left|a_{0}\right|<|a|<0,0<r \leq 1$, and all $0<p<\infty$.

It will be interesting to see if the approach in Theorem 2.1 can be extended to the univalent functions in $\mathcal{D}$. The result of this paper provides further evidence in favor of a conjecture made in [1]:

Conjecture $1 . \Lambda_{\Phi_{1}}$ attains its maximum on $\mathcal{B}$ at $B_{0}$.

\section{REFERENCES}

1. Valentin V. Andreev, and Alec Matheson, Extremal functions and the Chang-Marshall inequality, Pacific J. Math. 162 (1994), no. 2, 233-246. MR.1251899 (95f:30051)

2. Albert Baernstein, II, Integral means, univalent functions and circular symmetrization, Acta Math. 133 (1974), 139-169. MR0417406 (54:5456)

3. S.-Y. A. Chang, and D. E. Marshall, On a sharp inequality concerning the Dirichlet integral, Amer. J. Math. 107 (1985), no. 5, 1015-1033. MR0805803 (87a:30055)

4. Joseph Cima, and Alec Matheson, A nonlinear functional on the Dirichlet space, J. Math. Anal. Appl. 191 (1995), no. 2, 380-401. MR,1324020 (96g:46015) 
5. Peter L. Duren, Univalent functions, Grundlehren der Mathematischen Wissenschaften [Fundamental Principles of Mathematical Sciences], 259, Springer-Verlag, New York, 1983. MR0708494 (85j:30034)

6. Peter L. Duren, Theory of $H^{p}$ spaces, Pure and Applied Mathematics, 38, Academic Press, New York, 1970. MR0268655 (42:3552)

7. Matts Essén, Sharp estimates of uniform harmonic majorants in the plane, Ark. Mat. 25 (1987), no. 1, 15-28. MR0918377 (89b:30024)

8. Jason James, and Alec Matheson, (in preparation).

9. Donald E. Marshall, A new proof of a sharp inequality concerning the Dirichlet integral, Ark. Mat. 27 (1989), no. 1, 131-137. MR.1004727 (90h:30097)

10. Alec Matheson, and Alexander R. Pruss, Properties of extremal functions for some nonlinear functionals on Dirichlet spaces, Trans. Amer. Math. Soc. 348 (1996), no. 7, 2901-2930. MR1357401 (96j:30003)

Department of Mathematics, Lamar University, P. O. Box 10047, Beaumont, Texas 77710

E-mail address: andreev@math.lamar.edu 